\title{
Identification of Face across Random Motion Blur, Illumination and Pose
}

\author{
Shruti Sharma \\ Mtech scholar, \\ NRI Institute of information \\ science \& technology Bhopal
}

\author{
Brahmi Sharman \\ Asst. Prof., \\ $\mathrm{NRI}$ Institute of information \\ science \& technology Bhopal
}

\begin{abstract}
Presented strategies for obtaining face identification in the existence of blur are maintain the convolution model and can't handle non-uniform blurring things that regularly occur from tilt and rotary motion in hand-held cameras. This paper, include a trend to propose a method for face recognition within the occurrence of space-varying motion blur comprise of arbitrarily-shaped kernels. We have a tendency to model the blurred face as a rounded arrangement of geometrically remodel instance of the targeted gallery face, and show that the set of all images obtained by non-uniformly blurring a given image forms a convex set. We 1st propose a non uniform blur-robust algorithmic program by creating use of constriction on the camera movement. The frame is then extended to handle illumination discrepancy by. At last, we tend to plan a graceful expansion to also account for dissimilarity in pose.
\end{abstract}

\section{Keywords}

Face recognition, random blur, sparsity, illumination, poses.

\section{INTRODUCTION}

We recognized that the accuracy of face detection systems deteriorates quite fast in unconstrained settings [2]. This is capable of attributed to degradations arise since blur, changes during illumination, expression, pose, partial occlusions etc. Movement blur, in exacting, deserves extra ordinary attention due to the ubiquity of cell phone and hand-held imaging process. Dealing through camera quake is very significant problem because, while tripod obstructs mobility, tumbling the revelation time affects the image feature. Besides, in-built sensors like gyros and accelerometers. Contain their own boundaries in sensing the camera activity. In uncontrolled surroundings, clarification and pose may also differ, extra compounding the problem. The focus of this document is on developing an understanding that can know faces across nonuniform blur, and varying illumination and pose. Generally, blurring because of camera shake has been sculptured as convolution with a single blur kernel, and therefore the blur is known to be uniform across the image, On the other hand, it's space-variant blur that's encountered often in hand-held cameras. while techniques have been projected that tackle the restoration of non-uniform blur by local space-invariance approximation, current methods for image restoration have sculptured the motion-blurred image as average of protectively transformed images. Face recognition method that work with focused images have complexity when presented with blurred data. Advanced to face recognition from blur images can be largely classified into four categories. Deblurring-based on which the probe image is firstly deblur and then used for recognition. On the other hand, deblurring artifact is major source of error mainly for moderate to weighty blurs. (ii) Joint deblurring and recognition, the flipside of which is computational complexity. Deriving blur- invariant characteristic for recognition. But these all are efficient only for mild blurs. The direct recognition advance of and within which re-blurred versions from gallery are compared with the blurred probe image. It's essential to notice that all of the above approaches suppose a simple spaceinvariant blur model. For handling the illumination, there mainly two directions of pursuit depend on the 9D subspace model for face and extracting and identical illumination insensitive facial characteristic. Tan unite the strengths of the above two technique and suggest an integrated framework that comprise an initial illumination normalization step for face recognition under hard lighting conditions. A subspace educational approach using image gradient orientations for illumination and occlusion-robust face recognition has been proposed in. Practical face recognition algorithms must possess the capability to be aware of faces across reasonable variations in pose. Techniques for face recognition across pose can largely be categorized into $2 \mathrm{D}$ and $3 \mathrm{D}$ techniques. A good survey article on this subject can be found. It has been observed that since human faces have similar in general configuration, a relatively low dimensional subspace face is used to describe. Dimensionality decreasing subspace methods like Principle Component learning, Linear Discriminated learning and Independent Component Analysis has been proposed for the job of face recognition. These approaches can be categorized into either generative or discriminative techniques. One of the major benefits of using generative approaches is that they are known as less sensitive to noise as compared to discriminative approaches.

In recent years, (SR) theories and Compressed Sensing have come forward as powerful tools for efficiently processing data in modern ways. This has led to resurgence in interest in the main beliefs of Sparse Representation (SR) and Compressed Sensing (CS) for face recognition. Wright initiates an algorithm, called Sparse Representation based Classification, where the training face images are the dictionary and a novel test image is classify by pronouncement of its sparse representation with this dictionary. This attempt was later extended to handle illumination variations and misalignment. Also, Nagesh and Li presented an expression-invariant face recognition method using disseminated dense sensing and joint sparsity models. A face recognition technique depend on sparse representation for knowing 3D face meshes under expressions using small level geometric features was projected matching pursuit filters for face characteristic detection and recognition. The filters were designed in the course of a simultaneous decomposition of a training set into a 2D wavelet growth designed to differentiate among faces. It was shown that the resultant algorithm was robust to facial appearance and the close environment. There is a number of difficulties that face detection systems based on sparse representation must overcome. One is the designing algorithms that robust to vary in illumination; and second is 
that algorithms need to competently scale as the number of people enrolled in the scheme increases. In some of the above advanced, the challenge mentioned above are met by collecting a set of images of each one person that spans the space of expected variations in illumination. The SRC approach recognizes faces by resolving an optimization difficulty over the set of images enrolled into the database. This clarification trades robustness and size of the database adjacent to computational efficiency. In this document, we present an algorithm to execute face recognition diagonally varying illumination and pose depend on learning low sized class specific dictionaries1. Our process consists of two main stages. In first stage, given training samples from every class, class specific dictionaries are trained with some set number of atoms 2 . In second stage, a novel test face image is expected onto the span of the atoms in each well-read dictionary. The residual vectors after used for classification. in addition, assuming the Lambertian reflectance model for surface of a face, we incorporate a relighting approach inside our framework so that we are able to add many elements to gallery and robustness to the illumination and pose changes can be recognize. In this setting, as will be apparent, our technique has the capability to identify faces even when only a single or a a small number of images are make available for training.

\section{LITERATURE SURVEY}

Abhijith Punnappurath et.al. [1] "Face Recognition across Non Uniform movement Blur, Illumination, and Pose" presented, technique can't handle non-uniform blurring situations that often arise from rotations and tilts in hand-held cameras. In this document, we propose a technique for face identification in the existence of space-varying activity blur consist of arbitrarily-shaped kernel. We mold the blurred face resembling a convex combination of geometrically changed instances of the focused portico face, and make clear that the set of all the images achieve by non-uniformly blurring a specified image forms a convex set. Firstly we propose a non uniform blur-robust algorithm by making using of the supposition of a sparse camera trajectory in the camera movement space to construct an energy function with 11-norm limitation on the camera shift. The structure is then extended to handle illumination dissimilarity by exploiting the fact that the set of all images get from a face image by non-uniform blurring and changing the illumination forms a bi-convex set. In Result, we suggest a well-designed extension account for variation in pose. We proposed a methodology to execute face recognition under the joint effects of non-uniform blur, and pose, illumination. We show that the set of images get by nonuniformly blurring a given image which using the TSF model is convex set specified by the convex hull of distorted versions of the image. Capitalizing on this outcome we initially planned a non-uniform motion blur-robust face recognition algorithmic rule NU-MOB. We have a tendency to then show that the set of all images get from a given image by non-uniform blurring and alter in illumination forms a biconvex set, and used this outcome to develop our non-uniform movement blur and illumination-robust algorithm rule MOBIL. We then complete the likely of MOBIL to handle non-frontal face by transform the gallery to new pose. We establish the supremacy of this technique called MOBILAP over modern techniques. Wide experiments were known on synthetic as well as real face data. The restriction of our approach is that important occlusions and large change in facial expressions can't be handled.
Rob Fergus et. al. [2] "Removing Camera Shake from a solo Photograph" Camera shake for the duration of exposure leads to object image blur and ruins many photographs. Conservative blind deconvolution methods normally presume frequency-domain limitation on images, or excessively simplified parametric forms for the movement path during camera shake. Actual camera motions can follow complicated paths, and a spatial domain prior can improved maintain visually salient image characteristics. We bring in a method to remove the effects of camera shake from badly blurred images. The technique assumes a uniform camera blur over the image and insignificant in-plane camera rotation. In sort to guess the blur from camera shake, the user must specify an image area with no saturation effects. We explain results for a range of digital photographs taken from personal photo album. There are a several common photographic effects that we do not clearly model, including object motion, saturation, and compression artifacts. Including these factors into our model be supposed to improve robustness. Currently we suppose images to have a linear tone scale, once the gamma improvement has been eliminated. However, cameras typically have a slight sigmoid shape to their tone response curve, so as to expand their energetic range. Ideally, this nonlinearity would be disinterested, perhaps by estimating it during inference or by determining the curve from a sequence of bracketed exposures.

Qi Shan et. al. [3] "High-quality Motion Deblurring from a single Image" We present a new algorithm for removing motion blur from a single image. Our method computes a deblurred image with a combined probabilistic model of both blur kernel estimation and unblurs image restoration. We present an examination of the causes of common artifacts found in current deblurring methods, and then introduces several novel conditions inside this probabilistic model that are inspired by our analysis. These terms include a model of the spatial randomness of noise in the blur image, as well a novel local smoothness earlier that reduces ringing artifacts by constraining contrast in the unblur image wherever the blurred image exhibits low contrast. Lastly, we describe a wellorganized optimization scheme that exchange between blur kernel estimation and unblurred image restoration until junction. As a outcome of these steps, we are able to produce high quality deblurred outcome in low working out time. We are even capable to produce results of similar quality to method that need additional input images outside a single blurry photograph, and to technique that require additional hardware. We have projected a novel image deconvolution technique to eliminate camera movement blur from a single image by decrease errors caused by inexact blur kernel estimation and image noise. We introduce a combined framework to solve both blind and non blind deconvolution troubles. Our major assistance is an effective model for image clatter that accounts for its spatial allocation, and a local prior to restrain ringing artifacts. These two models interrelate with each other to advance unblurred image assessment even with a very simple and imprecise initial kernel after our superior optimization process is functional.

Raghuraman Gopalan et.al. [4] "A Blur-robust Descriptor with application to Face Recognition" perceptive the cause of blur is an significant problem in unconstrained visual analysis. We deal with this problem in the context of image-based identification, by a fusion of image-formation models, and disparity geometric tools. At First, we discuss the space spanned by blur versions of an image and then in certain supposition, present a differential geometric examination of that space. completely, we create a subspace resultant from 
convolution of an image with a entire set of ortho normal basis functions of a pre-define maximum size, and show that the equivalent subspaces created from a fresh image and its blurred versions are equivalent under the ideal case of zero noise, and some suppositions on the property of blur kernels. Then we study the realistic utility of this subspace demonstration for the problem of direct identification of blurred faces, by screening the subspaces as point on the Grossmann manifold and present techniques to perform identification for condition where the blur is both spatially varying and homogenous. We empirically examine the effect of noise, as well as the existence of other facial variation in between the gallery and probe images, and offer comparisons with offered approaches on ordinary datasets. We explain that the subspace resultant from convolutions of an image with a total set of orthonormal basis functions that could characterize the blur kernel is invariant to blur under some statement, and it can account for more common classes of blur unlike other invariants. We then calculated the utility of this invariant for the problem of direct identification of faces, using procedure that account for their underlying non-Euclidean geometry, and observed an enhanced performance over added presented deblurring-based and invariant-based approaches. From the point of sight of performing robust face identification under unimpeded settings, it is attractive to study the incorporation of explicit formulations of other facial variations such as lighting and pose, by this blur-invariant.

Vishal M. Patel et. al. [5] "Dictionary-based Face identification under uneven Lighting and Pose" a face recognition algorithm depend on simultaneous sparse estimation under varying illumination and pose. A dictionary is well-read for each class based on given training model which reduced the demonstration error with a sparseness constraint. A new test image is expected onto the span of the atoms in each well-read dictionary. The resultant residual vectors are then use for arrangement. To hold the variations in lighting circumstances and pose, an image relighting method based on pose-robust albedo assessment is used to generate multiple frontal images of the identical person with uneven lighting. As an effect, the proposed algorithm has the capability to recognize human faces with high accurateness even when only a single or a very few images per person are offered for training. The efficiency of the projected method is demonstrated using openly available databases and it is made known that this technique is well-organized and can perform extensively better than many competitive face identification algorithms. We have projected a face recognition algorithm based on dictionary learning technique that is robust to change in lighting and pose. This brings about using a relighting advance based on robust albedo judgment. Different experiments on popular face identification data sets have shown that our technique is proficient and can execute significantly better than many competitive face identification algorithms.

\section{METHOD}

Images of the same person can differ significantly due to variations in illumination conditions. Therefore, the performance of most accessible face recognition algorithms is highly sensitive to illumination deviation. In this section, we initiate a relighting technique to deal with this illumination difficulty. The thought is to capture the illumination circumstances that may occur in the experiment sample in the training samples

\subsection{Albedo estimation}

Presuming the Lambert and reflectance model for the facial surface, one is able to relate the surface normals, albedo and the strength image by an image creation model. The diffused part of the surface reflection is specified by

$$
x_{i, j}=\rho_{i, j} \max \left(n_{i, j}^{T} s, 0\right)
$$

Where $x_{i, j}$ is the pixel intensity at the position (i,j), s is light source direction, $\rho_{\mathrm{i}, \mathrm{j}}$ is surface albedo at position $(\mathrm{i}, \mathrm{j})$, where $\mathrm{n}_{\mathrm{i}, \mathrm{j}}$ is the surface normal of the equivalent surface point and 1 $\leq \mathrm{i} \leq 1,1 \leq \mathrm{j} \leq \mathrm{q}$. The max function in (10) account for the creation of attached shadows. Ignore the close shadows, (1) can be linearized such as

$$
\begin{aligned}
x_{i, j} & =\rho_{i, j} \max \left(n_{i, j}^{T} s, 0\right) \\
& \approx \rho_{i, j} n_{i, j}^{T} s
\end{aligned}
$$

Let $n_{i, j}^{(0)}$ and ${ }^{(0)}$ be the primary values of the surface normal and illumination direction. These initial values be able to be domain needy average values. The Lambertian supposition impose the following limitation on the initial albedo

$\rho_{i, j}^{(0)}=\frac{x_{i, j}}{n_{i, j}^{(0)} \cdot s^{(0)}}$

Where '.' denotes the typical dot product operation. Using (2), equation (3) can be rewrite as

$$
\begin{aligned}
\rho_{i, j}^{(0)}=\rho_{i, j} \frac{n_{i, j} \cdot s}{n_{i, j}^{(0)} \cdot s^{(0)}} & =\rho_{i, j}+\frac{n_{i, j} \cdot s-n_{i, j}^{(0)} \cdot s^{(0)}}{n_{i, j}^{(0)} \cdot s^{(0)}} \rho_{i, j} \\
& =\rho_{i, j}+\omega_{i, j}
\end{aligned}
$$

Where

$$
\omega_{i, j}=\frac{n_{i, j} \cdot s-n_{i, j}^{(0)} \cdot s^{(0)}}{n_{i, j}^{(0)} \cdot s^{(0)}} \rho_{i, j}
$$

This can be view as a signal assessment problem where $\rho$ is the creative signal; $\rho(0)$ is the degraded signal. The signal dependent noise is $\omega$. By using this model, the albedo can be projected using the method of lowest mean squared error standard. Then, using the projected albedo map, one can produce new images for a given light source direction using the image creation model in (1). This be able to be done by combine the estimated albedo map and light source direction with average facial details.

\subsection{Image relighting}

It has been originate that the set of images in all possible illumination circumstances can be well approximated by a 9 dimensional linear subspace. Depend on this result, Lee showed that there exist a pattern of 9 light source directions such that the subspace created by the images taken under these nine sources is efficient for recognize faces under a extensive range of lighting conditions. The nine prespecified light source guidelines are given by

$$
\begin{aligned}
& \varphi=\{0,49,-68,73,77,-84,-84,82,-50\}^{\circ} \\
& \theta=\{0,17,0,-18,37,47,-47,-56,-84\}^{\circ} .
\end{aligned}
$$

Therefore, the image pattern equation can be re-written as

$$
x=\sum_{i=1}^{9} a_{i} x_{i}
$$

Where $x_{i}=\rho_{\max }\left(n^{T} s_{i}, 0\right)$ and $\left\{s_{1}, \cdots, s_{9}\right\}$ are the pre specific 
illumination directions. To differentiate the set of images under a variety of illumination conditions, one can produce images under the nine pre-specified illumination directions and make use of them in the gallery. By generating multiple face images with diverse lighting from a single face image, one can attain good identification accuracy even when only a solo or a very few images are offered for training.

\subsection{Pose-robust albedo estimation}

The technique offered above can be generalized such that it can handle pose variation. Let $\bar{n}_{i, j}, \bar{s}$ and $\bar{\theta}$ be a few initial estimates of the surface normals, illumination direction and preliminary estimate of surface normals in $\operatorname{pose} \theta$, correspondingly. Then, the initial albedo at pixel $(i, j)$ can be represent the initial estimation of surface normals in pose. by means of this model, we can re-formulate the difficulty of recovering albedo as a signal assessment problem. Using arguments similar to equation (12), we get the subsequent formulation for the albedo assessment problem in the presence of pose

$$
\rho_{i, j}=\rho_{i, j} h_{i, j}+\omega_{i, j},
$$

Where $\rho_{\mathrm{i}, \mathrm{j}}$ is the factual albedo and $-\rho_{\mathrm{i}, \mathrm{j}}$ is the degraded albedo. In the case when the pose is known precisely, and $\mathrm{h}_{\mathrm{i}, \mathrm{j}}$ $=1$. Therefore, this can be view as a simplification of (4) in the case of indefinite pose. Using this model, a stochastic filtering structure was recently presented in to estimation the albedo from a single non-frontal face image. One time pose and illumination have been normalized, individual can use the relighting technique described in the earlier section to generate multiple frontal images with diverse lighting to achieve illumination and pose-robust recognition. Some example of pose normalized images using this technique. We review our dictionary-based face recognition algorithm. Note that a K-SVD based face identification algorithm was recently planned in but we differ from this work in a only some key areas. Distinct we do not take discriminative approach to face identification. Our process is a reconstructive approach to discrimination and does not need multiple images to be available. Dissimilarity is that our algorithm has the capability to recognize and reject non-face images.

\section{CONCLUSION}

We projected a methodology to perform face recognition under the mutual effects of non-uniform blur, illumination, and pose. We show that the set of all images obtain by nonuniformly blurring a known image by using the TSF model is a convex set given by the convex hull of distorted versions of the image. Capitalizing on this result, we primarily proposed a non-uniform motion blur-robust face identification algorithm NU-MOB. We then show that the set of all images obtain from a given image by non-uniform blurring and change in illumination forms a bi-convex set, and used this effect to develop our non-uniform motion blur and illumination-robust algorithm MOBIL. Then we complete the potential of MOBIL to grip even non-frontal faces by transforming the gallery to a new pose. We recognized the superiority of this method called MOBILAP over contemporary technique. Widespread experiments were known on synthetic as well as real face data. The constraint of our approach is that important occlusions and large changes in facial expressions can't be handled.

\section{REFERENCES}

[1] Abhijith Punnappurath, Ambasamudram Narayanan Rajagopalan, Sima Taheri, Rama Chellappa "Face Recognition Across Non-Uniform Motion Blur, Illumination, and Pose" ieee transactions on image processing, vol. 24, no. 7, july 2015

[2] R. Fergus, B. Singh, A. Hertzmann, S. T. Roweis, and W. T. Freeman,"Removing camera shake from a single photograph" ACM Trans. Graph., vol. 25, no. 3, pp. 787-794, Jul. 2006.

[3] Q. Shan, J. Jia, and A. Agarwala, "High-quality motion deblurring from a single image" ACM Trans. Graph., vol. 27, no. 3, pp. 73:1-73:10, Aug. 2008.

[4] R. Gopalan, S. Taheri, P. Turaga, and R. Chellappa, “A blur-robust descriptor with applications to face recognition" IEEE Trans. Pattern Anal. Mach. Intell., vol. 34, no. 6, pp. 1220-1226, Jun. 2012.

[5] V. M. Patel, T. Wu, S. Biswas, P. J. Phillips, and R. Chellappa, "Dictionary-based face recognition under variable lighting and pose" IEEE Trans. Inf. Forensics Security, vol. 7, no. 3, pp. 954-965, Jun. 2012.

[6] S. Cho, Y. Matsushita, and S. Lee, "Removing nonuniform motion blur from images" in Proc. Int. Conf. Comput. Vis., Oct. 2007, pp. 1-8.

[7] Y.-W. Tai, P. Tan, and M. S. Brown, "Richardson-Lucy deblurring for scenes under a projective motion path" IEEE Trans. Pattern Anal. Mach. Intell., vol. 33, no. 8, pp. 1603-1618, Aug. 2011.

[8] O.Whyte, J. Sivic, A. Zisserman, and J. Ponce, "Nonuniform deblurring for shaken images" Int. J. Comput. Vis., vol. 98, no. 2, pp. 168-186, 2012.

[9] A. Gupta, N. Joshi, L. Zitnick, M. Cohen, and B. Curless, "Single image deblurring using motion density functions" in Proc. Eur. Conf. Comput. Vis., 2010, pp. $171-184$

[10] Z. $\mathrm{Hu}$ and M.-H. Yang, "Fast non-uniform deblurring using constrained camera pose subspace" in Proc. Brit. Mach. Vis. Conf., 2012, pp. 1-11. 